\title{
Yaşlı kalça kırıklarında AO prensiplerine göre plak-vida uygulaması
}

\section{Plate-screw fixation for elderly hip fractures according to $\mathrm{AO}$ principles}

\author{
Ufuk Özkaya ${ }^{1}$, Arsen Arsenishvili \\ ${ }^{1}$ Bahçelievler Memorial Hastanesi, Ortopedi ve Travmatoloji Kliniği, İstanbul \\ ${ }^{2}$ Savunma Bakanlığı, Merkezi Askeri Hastanesi, Gori, Gürcistan
}

\begin{abstract}
Yaşlılarda kalça kırıkları, komplikasyon ve mortalite oranları yüksek olan problemli kırıklardır. Femur boyun kırıklarında öncelikle artroplasti seçenekleri tercih edilir. Stabil intertrokanterik kırıklarda hem kanal içi (intramedüller), hem kanal dışı (ekstramedüller) implantlar başarılı bir şekilde kullanılabilirken, instabil intertrokanterik ve subtrokanterik kırıklarda kanal içi çivileme tekniği biyomekanik üstünlüğü nedeniyle daha çok yaygınlaşmıştır. Ancak ekstramedüller implantlarla, yani plak vida ile osteosentez ile tespit yöntemi, intramedüller tespitin kullanılmasının yol açabileceği komplikasyonlardan kaçınılması gereken durumlarda, minimal invaziv cerrahi kullanılması şartıyla bir alternatif tedavi yöntemi olabilir. Osteoporoz, kırık tespitinin başarısız olmasına veya redüksiyon kaybına yol açabilir. Kilitli plaklar özellikle osteoporotik kırık tespitinde kullanılması için geliştirilmiştir. Kırık redüksiyonuna gereken özenin gösterilmemesi, yaşlılardaki kalça kırıklarının osteosentezinde başarısızlığın en sık nedenidir.
\end{abstract}

Anahtar sözcülkler: osteoporoz; kırık; internal tespit; plak ile osteosentez; proksimal femur kırıkları; ters LISS
Geriatric hip fractures are problematic fractures with their high complication and mortality rates. Arthroplasty is preferred primarily in the surgical management of femoral neck fractures. While stable intertrochanteric fractures can successfully be managed with both intramedullary and extramedullary fixation methods, intramedullary fixation has been more widely accepted in the management of unstable intertrochanteric and subtrochanteric fractures due to its biomechanical advantages. However, extramedullary fixation can be an alternative option if minimally invasive surgery is used to avoid complications of intramedullary fixation in selected cases. Osteoporosis may cause both loss of initial reduction and failure of the implant construct. Locked plate technology has been developed as a solution to be used in osteoporotic geriatric hip fracture fixation. Failure of obtaining good reduction of the fracture is the main reason in poor results after surgical management of this group of patients.

Key words: osteoporosis; fracture; internal fixation; plate osteosynthesis; proximal femoral fractures; reverse LISS

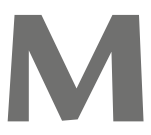

odern tıptaki ve yaşam kalitesindeki artışlara paralel olarak toplumdaki geriatrik (yaşı) nüfus artmakta, bunun sonucunda geriatrik kalça kırıklarının görülme sıklığı da artmaktadır. Uluslararası Osteoporoz Vakfi'nın yaptığı bir çalışmaya göre osteoporoz nedenli kalça kırıklarının görülme sıklığı yıllık 5.7 milyon kadardır ve 2050 yılına kadar bu sayının 20 milyona ulaşması beklenmektedir. ${ }^{[1]}$ Kalça kırıkları anatomik bölgelerine göre boyun ve baş kırıkları, intertrokanterik kırıklar ve subtrokanterik kırıklar olarak adlandırılmaktadır.

Geriatrik popülasyonda kalça kırıklarında başarıya doğrudan etki eden birkaç faktör vardır. Kemiğin kalitesi, kırı̆̆ın parçalanma miktarı ve dolayısıyla stabilitesi ve hastaya eşlik eden hastalıklar cerrahın elinde olmayan, değiş̧iremeyeceği faktörlerdir. Ancak kırığın redüksiyon kalitesi, kullanılan implant tipi, cerrahın tecrübesi, hastane ve ameliyathane şartları doğrudan cerraha bağlı değişkenlerdir.

Geriatrik popülasyonda ayrışmamış boyun kırıklarında dinamik kalça vidası (DHS) ve/veya kanüllü vida ile osteosentez kullanılabileceği bildirilmişse de ayrışmış femur boyun kırıklarının tedavisinde osteosentezin sonuçları genellikle çok başarılı değildir (Şekil 1).

Bu nedenle geriatrik femur boyun kırıklarında parsiyel ya da total artroplasti ağırlıklı olarak tercih edilen tedavi yöntemidir. ${ }^{[2,3]}$

- Prof. Dr. Ufuk Özkaya, Bahçelievler Memorial Hastanesi, Bahçelievler Merkez, Eski Londra Asf. Cd. No: 227, 34180 Bahçelievler, İstanbul

Tel: 0532 - 2652212 e-posta: ufukozkaya2006@gmail.com

- Geliștarihi: 1 Șubat 2021 Kabul tarihi: 10 Subat 2021

ORCID iD: Ufuk Özkaya, 0000-0003-0792-206X • Arsen Arsenishvili, 0000-0001-8315-2285 


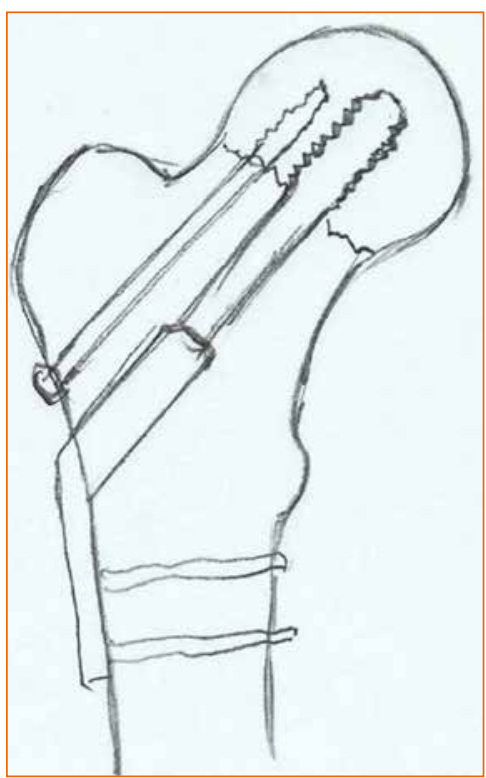

Şekil 1. Femur boyun kırığında kanüllü vida ve DHS'nin birlikte kullanılması.

Intertrokanterik ve hatta bazen subtrokanterik kırıkların tedavisinde artroplasti bazı yazarlarca önerilmiş olsa da, ${ }^{[4,5]} \mathrm{AO}$ prensiplerine göre bu kırıkların tedavisinde intramedüller ve ekstramedüller osteosentez daha ön planda önerilmektedir. ${ }^{[6]}$ Stabil intertrokanterik kırıklarda DHS ile başarılı sonuçlar alınabilir. ${ }^{[7,8]}$ İnstabil intertrokanterik ve subtrokanterik kırıkların tedavisinde ise intramedüller çivileme tekniği, ekstramedüller implantlara karşı biyomekanik üstünlüğü ve minimal invaziv uygulanması nedeniyle AO tarafından ilk planda kullanılması tavsiye edilen tespit yöntemidir. ${ }^{[9-13]}$

Femurun öne eğimi (anterior femoral bowing) bazı geriatrik hastalarda artmış olabilir. Ameliyat öncesi planlamada bu durum gözden kaçırılırsa intramedüller çivileme esnasında tedavi kaynaklı (iyatrojenik) femur şaft (cisim) kırığı meydana gelebilir. ${ }^{[10]}$ Özellikle atipik femur kırıklarının intramedüller çivi ile tespiti esnasında da iyatrojenik femur şaft kırı̆̆ı gelişebilir. ${ }^{[14]} \mathrm{Bu}$ gibi durumlarda ekstramedüller tespit tercih edilebilir. Ekstramedüller tespitin endike olabileceği bir başka durum, kilitli intramedüller çivilemenin kullanılmasını engelleyen, önceden geçirilmiş uzun stemli revizyon diz artroplasti ameliyatı varlığıdır. ${ }^{[10,15,16]}$

Ekstramedüller implantlarla yani plak-vidalarla osteosentez yönteminde implant seçenekleri arasında paslanmaz çelik ya da titanyum olmak üzere, klasik $135^{\circ}$ 'lik DHS (Şekil 2, 3), $95^{\circ}$ lik dinamik kondiler vida (DCS) (Şekil 4), trokanterik stabilizasyon plağı, her üç plağın özellikle osteoporotik hastalarda

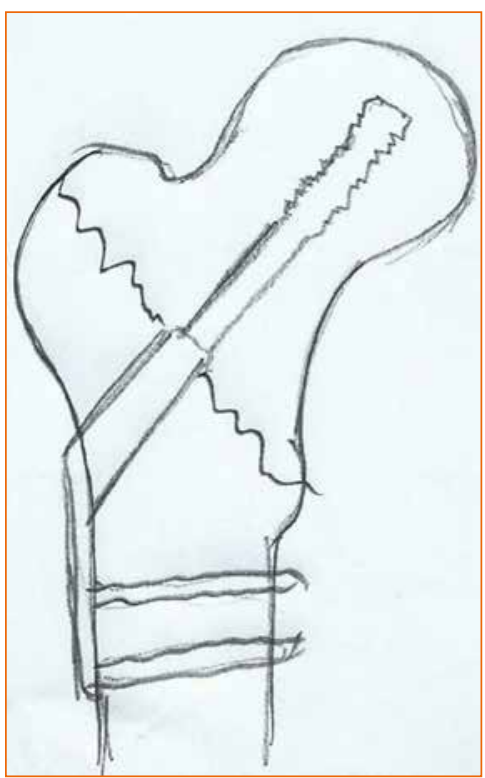

Şekil 2. İntertrokanterik femur kırığında $135^{\circ}$ DHS uygulaması.

avantaj sağlayan, kilitli vida tekniğinin kullanıldığı modifikasyonları, $95^{\circ}$ açılı kamalı plaklar (Şekil 5), proksimal femur kilitli plaklar (Şekil 6, 7) ve karşı taraf distal femur için dizayn edilen distal femur kilitli plaklarının ters çevrilerek kullanıldığı ters LISS (Less invasive stabilization system) (Şekil 8, 9, 10) kilitli plakları vardır. ${ }^{[17-19]}$

\section{Kemik Kalitesi}

Osteoporotik kırıklar, geriatrik popülasyonda sıktır. Kadınlarda özellikle menopoz sonrası dönemde görülme sıklığı artar. Osteoporozda tespit problemi başarılı sonuç için en büyük engellerden birisidir. Kilitli plaklar AO tarafindan osteoporoz gibi kemik kalitesi bozuk hastalarda çözüm amaçlı geliştirilmiş ve hızla yaygınlaşarak dünya genelinde kabul edilmiştir. ${ }^{[20,21]}$

Geriatrik kırıklarda osteoporoz nedeniyle ortaya çıkan tespit probleminin çözümü için önerilen bir diğer teknik, kırık sahasına değil ama vidaların osteoporotik kemiğe tutunmasını artırmak için kemik çimentosu kullanılmasıdır. ${ }^{[22]}$

\section{Kırığın Parçalanma Miktarı}

Stabil kırıklarda stabilitenin korunması, kortikal devamlılığın ne kadar sağlanabildiğine, yani redüksiyonun başarısına bağlıdır. İnstabil, ters oblik ya da çok parçalı pertrokanterik kırıklarda kortikal devamlılığın sağlanması zor ve hatta bazen imkânsızdır. İmplanta binen yük çok fazla olacağı için çok daha dayanıklı olmalıdır. Kemik-implant stresini ve implanta binen yükü 


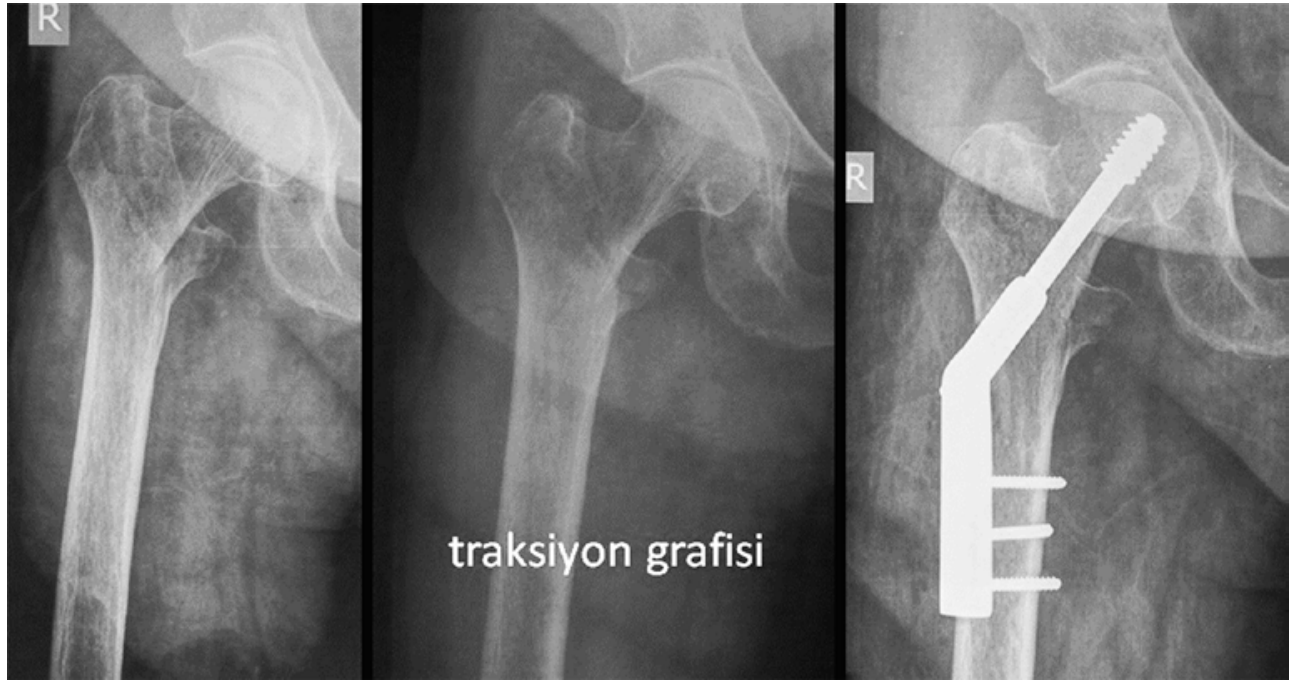

Şekil 3. $135^{\circ}$ DHS kullanarak 85 yaşındaki hastanın stabil intertrokanterik kırığının tespiti.

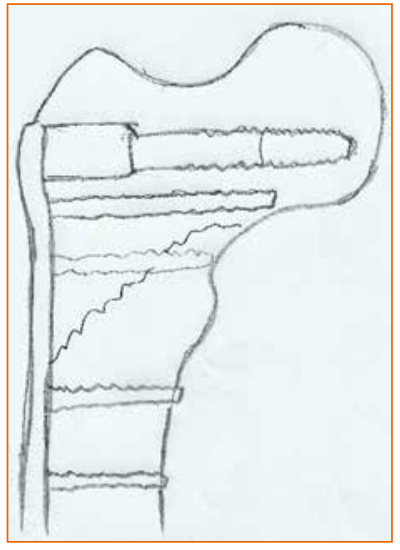

Şekil 4. $95^{\circ}$ dinamik kondiler vida uygulaması.

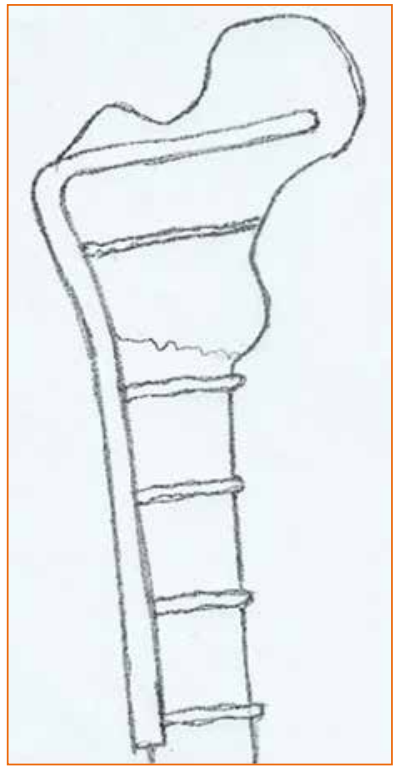

Şekil 5. $95^{\circ} \mathrm{AO}$ kamalı plak uygulaması.
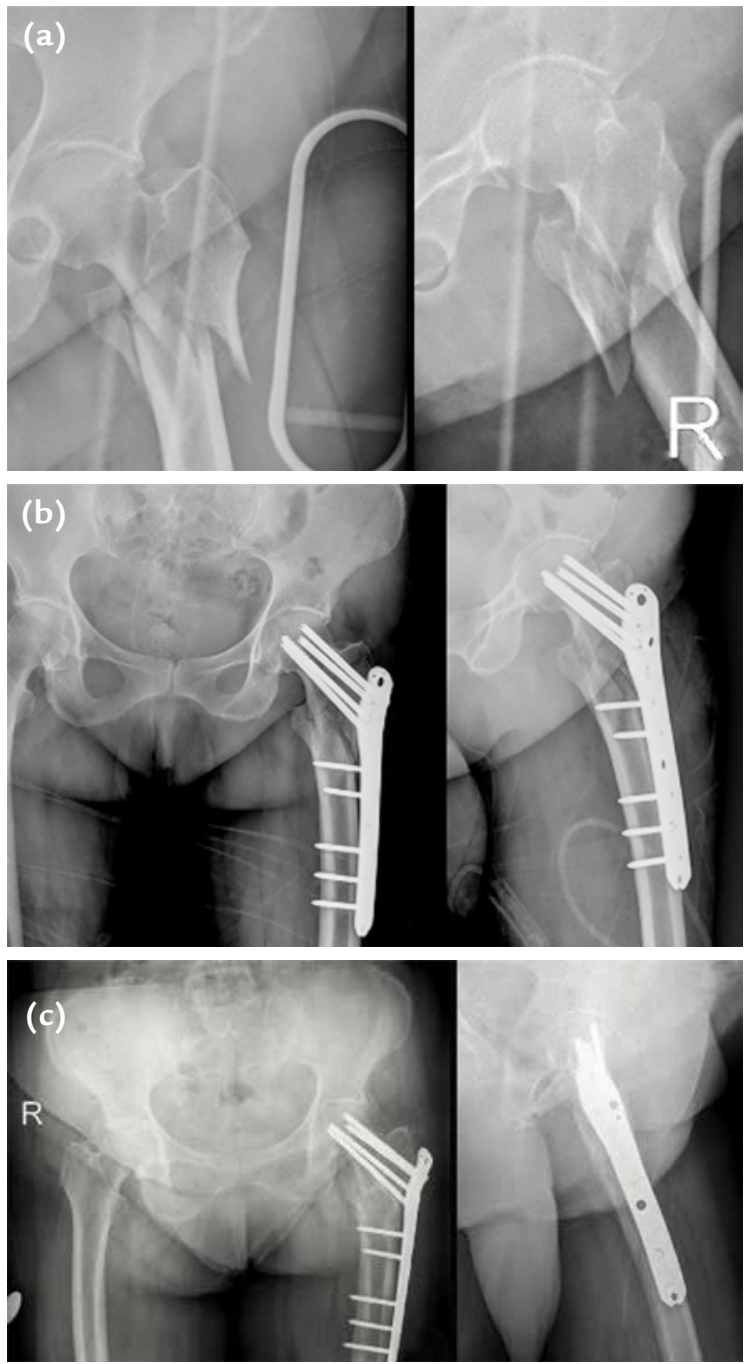

Şekil 7. a-c. Proksimal femur kilitli plak kullanarak 78 yaşındaki hastanın instabil intertrokanterik kırığının tespiti; ameliyat öncesi (a) ile sonrası erken (b) ve geç (c) dönem grafileri. 


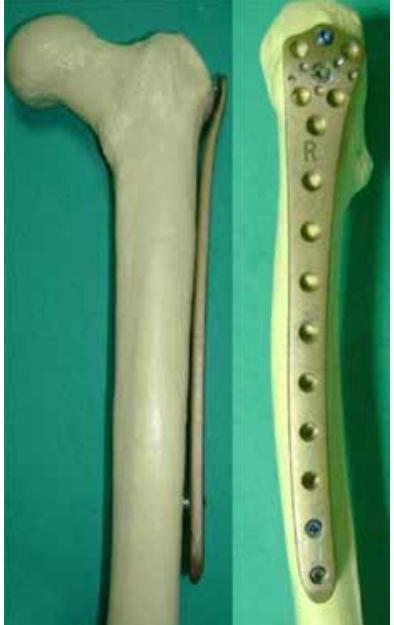

Şekil 8. Ters LISS plağı.
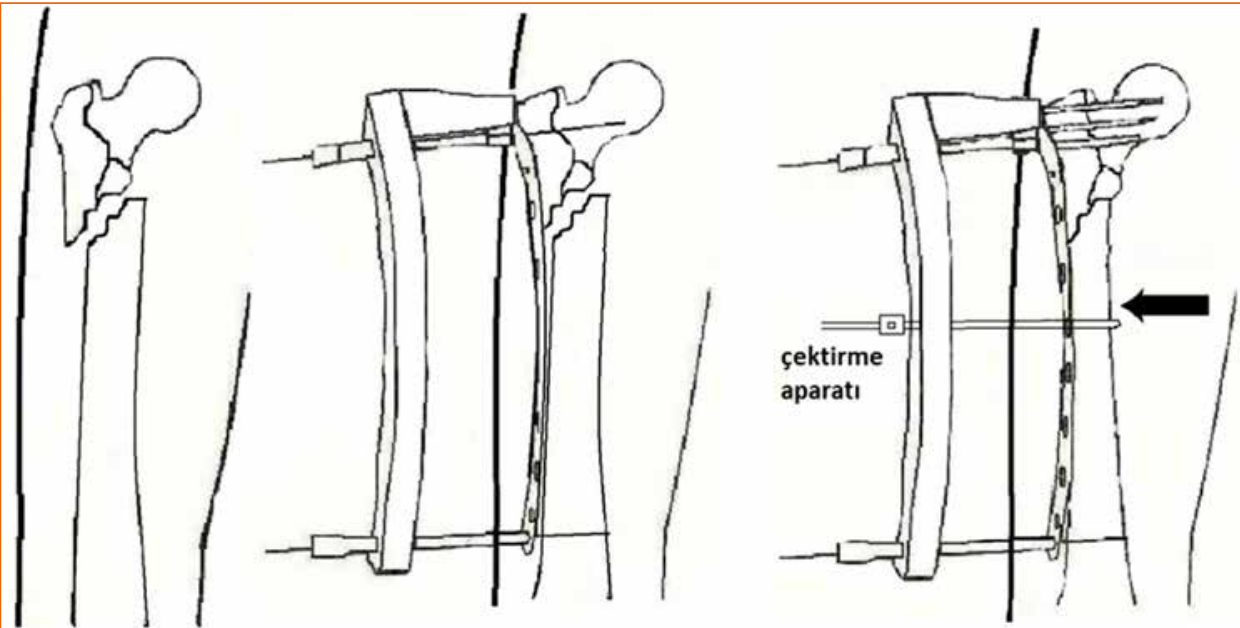

Şekil 9. Ters LISS plağı; cerrahi teknik.
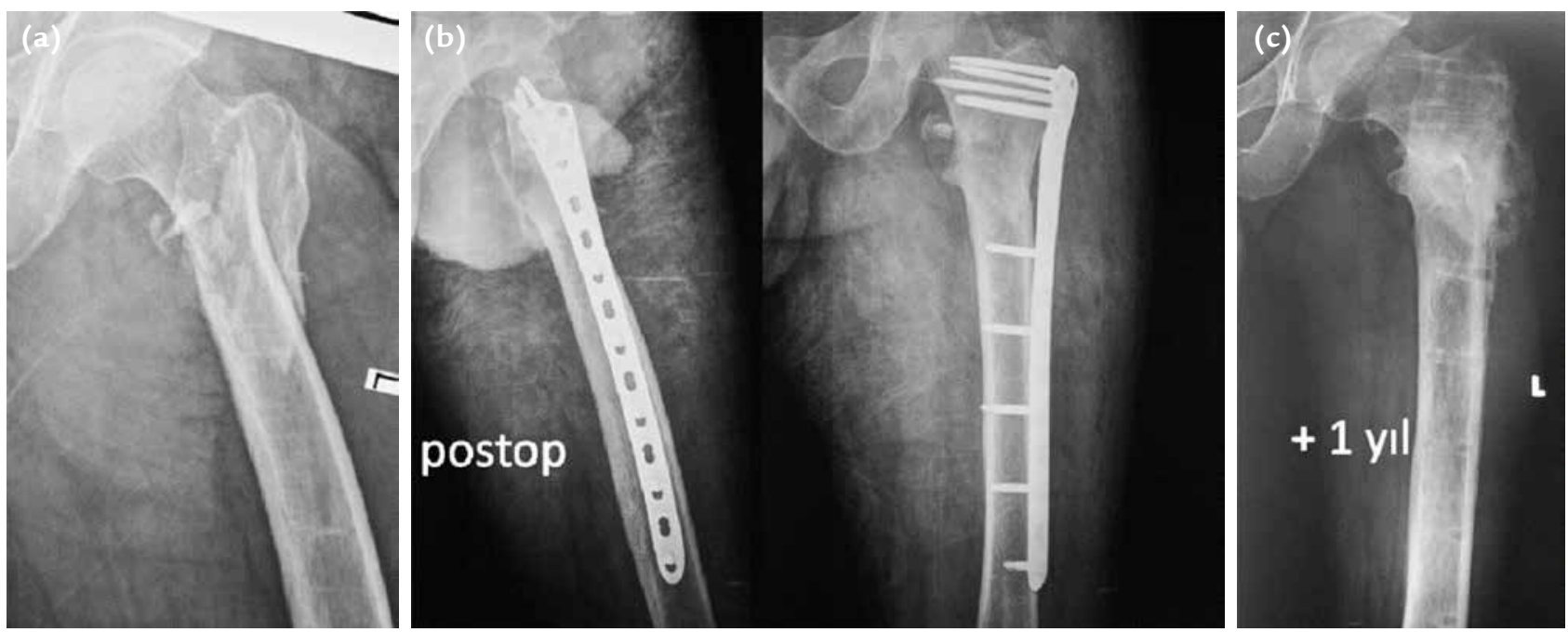

Şekil 10. a-c. Ters LISS plağı kullanılarak 80 yaşındaki hastanın instabil intertrokanterik kırığının tespiti; ameliyat öncesi (a), sonrası (b) ve plak çıkartıldıktan sonraki (c) grafileri.

azaltmak, böylece fragmanlar arası kompresyonu sağlayabilmek için proksimal fragmanın hafif valgusta redükte edilmesi önerilmektedir. ${ }^{[23]}$

\section{Redüksiyon}

Redüksiyon kalitesi, cerrahın elinde olan değişkenlerden en önemlisidir. Kalça kırıklarının tedavisinde ameliyatın başarısına doğrudan etki eden en önemli etken, redüksiyonun kalitesidir. ${ }^{[24]}$ Redüksiyonda doğrudan veya dolaylı redüksiyon yöntemleri kullanılabilir. Varus deformitesi tüm kalça kırıklarında en sıklıkla karşılaşılan deformitedir ve asla kabul edilmemesi gerekir (Şekil 11).

İntertrokanterik kırıklarda varus deformitesinin yanı sıra kısalık ve dış rotasyon deformiteleri birlikte

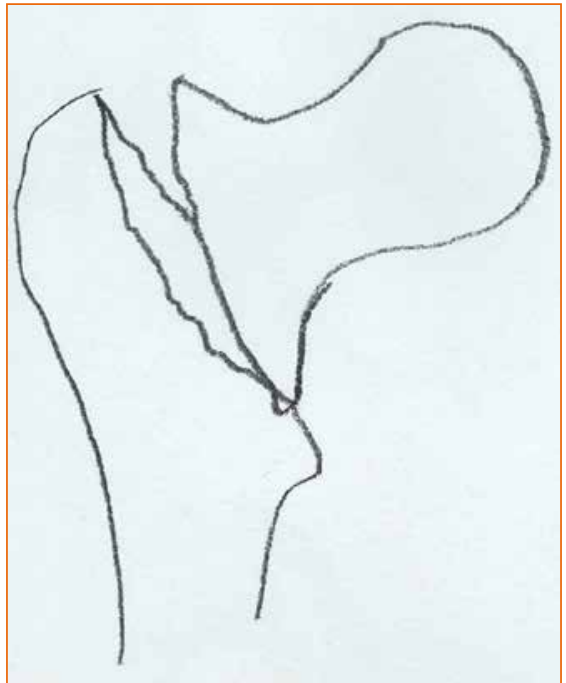

Şekil 11. Intertrokanterik kırıkta varus deformitesi. 


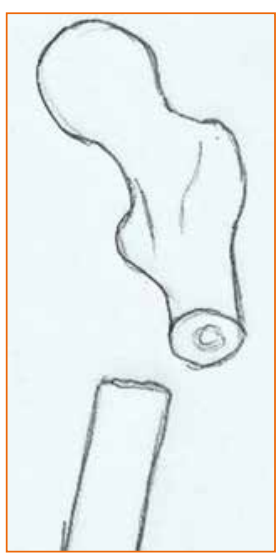

Sekil 12. Subtrokanterik kırıkta tipik deformite.

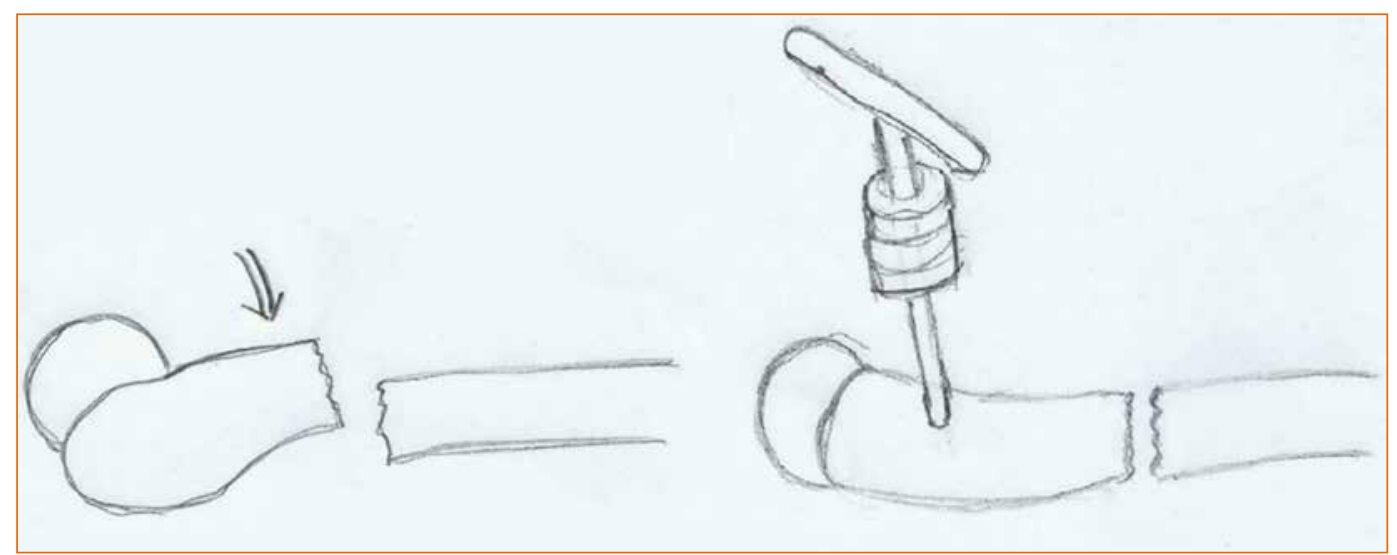

Şekil 13. Joystick olarak kullanılan Schanz vidası ile proksimal fragman fleksiyonunun redüksiyonu.

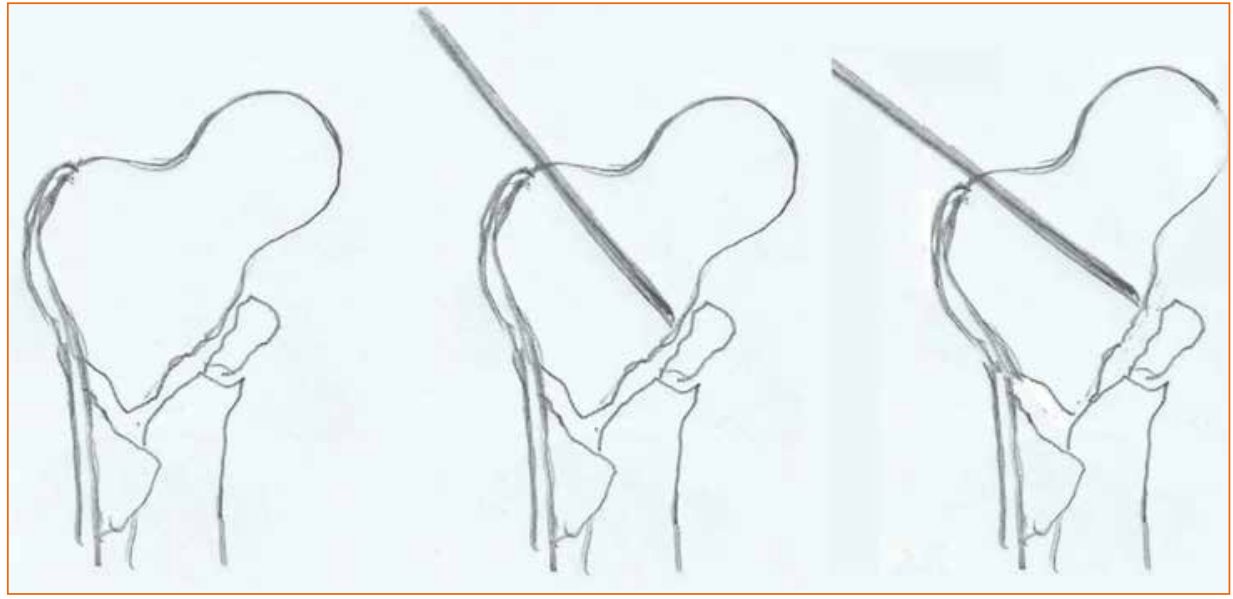

Şekil 14. Joystick olarak kullanılan Schanz vidası ile proksimal fragman varus deformitesinin redüksiyonu.

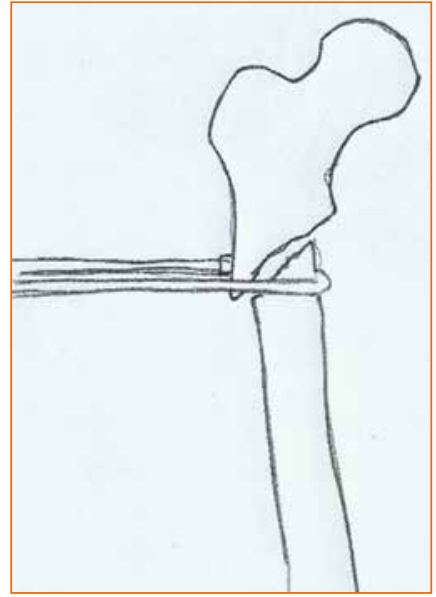

Şekil 15. Colinear klemp ile redüksiyon. görülür. Redüksiyonu sağlamak için cerrahın tercihine bağlı olarak traksiyon masası, asistan yardımlı elle traksiyon, dışarıdan çekiç veya benzeri sert bir aparat kullanarak ittirme gibi kapalı, joystick Schanz vidası, çengel, elevatör gibi invaziv doğrudan ve dolaylı redüksiyon yöntemleri kullanılabilir. ${ }^{[25]}$

Subtrokanterik kırıklarda ise proksimal fragman varus deformitesinin yanı sıra gluteus medius ve minimus kaslarının çekmesi ile abduksiyonda, iliopsoas kasının çekmesiyle fleksiyon ve dış rotasyonda, adduktorların distal fragmanı mediale çekmesi nedeni ile ayrışmış durumda durmaktadır (Şekil 12).

Kırığı deforme edici kaslar varusa yol açabildiği gibi, kullanılan implantlar da varus deformitesine yol açabilir. Başarılı bir redüksiyon sağlanmasında kırığı deforme edici mekanizmaların anlaşılması ve deforme edici güçleri yenici manevraların yapılması şarttır. Proksimal fragmanın fleksiyon deformitesi, proksimal fragmana yerleştirilecek bir Schanz vidasının joystick manevrası ile düzeltilebilir (Şekil 13).

Proksimal fragmanın varus deformitesi, proksimal fragmana yerleştirilecek bir Schanz vidasının joystick manevrası ile düzeltilebilir (Şekil 14).

Özellikle subtrokanterik kırıkların redüksiyonunda kapalı dolaylı redüksiyon manevraları yetersiz kaldığında doğrudan redüksiyon gerekebilir. Bu durumda Colinear klemp gibi özel cihazların kullanılması, minimal kesi ile yumuşak doku hasarına yol açmadan kırığın redükte edilmesinde çok büyük kolaylık sağlar (Şekil 15, 16).

\section{Minimal İnvaziv Cerrahi}

Sadece kalça kırıklarının değil, tüm anatomik bölgelerin cerrahisinde hedeflenen redüksiyon ve tespit, olabilecek 

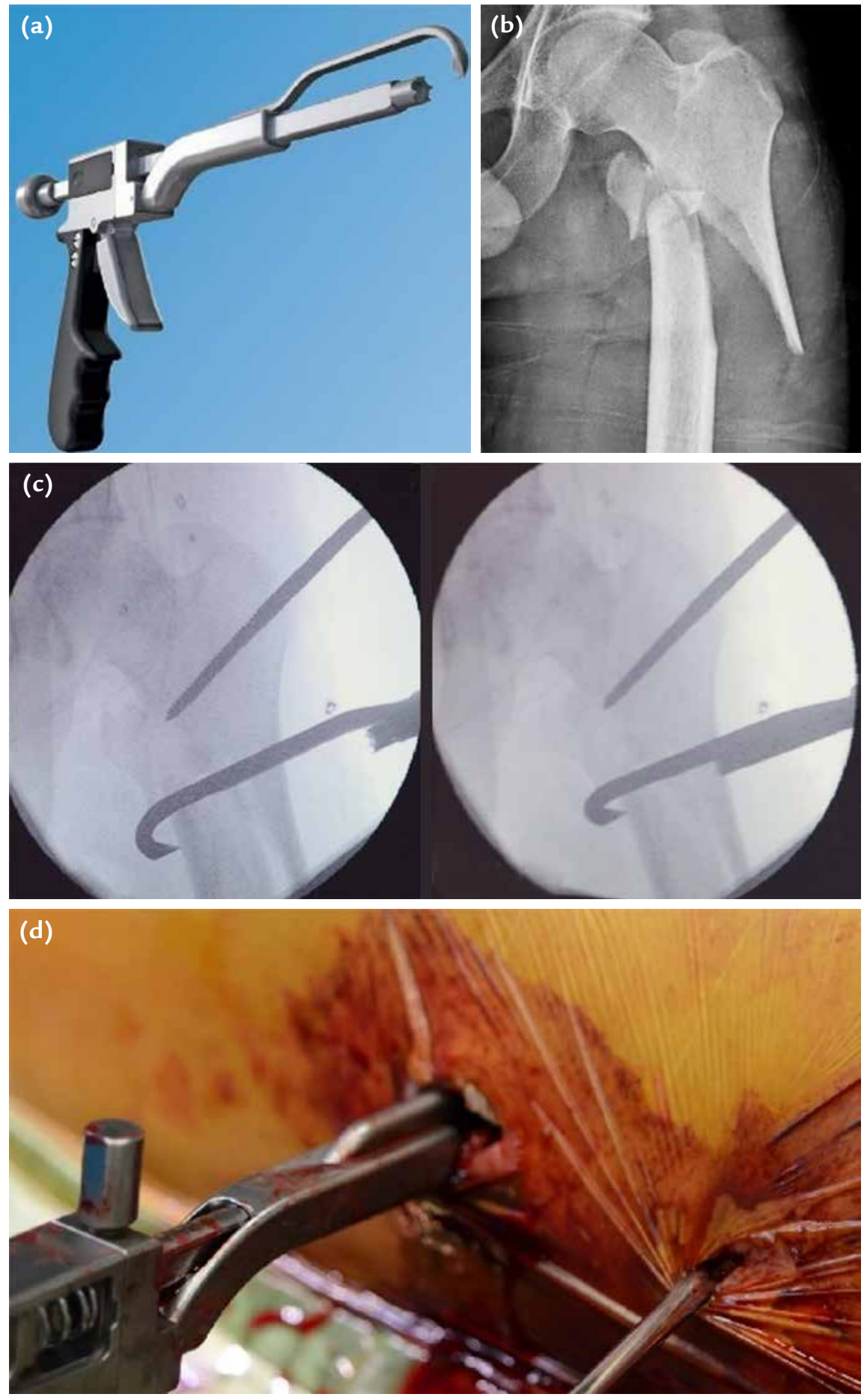

Şekil 16. a-d. Colinear klemp ile introperatif minimal invaziv kırık redüksiyonu; Colinear klemp (a), ameliyat öncesi ön-arka (AP) grafi (b), ameliyat esnasında uygulanan redüksiyonun skopi görüntüsü (c) ve ciltten yapılan minimal kesiden Colinear klempin uygulanması (d). en az ek cerrahi hasar verilerek yapılmalıdır. Kırık esnasında zaten hasarlanan yumuşak dokuyu mümkün olduğunca korumak esastır. Ancak, olabildiğince az travmatik cerrahi kullanılarak stabil osteosentez yapılması, bunların sonucunda da erken hareket ve mobilizasyona izin verilebilmesi gereklidir (Şekil 17). ${ }^{[17,18]}$
AO'nun tüm travmatolojide temel prensipleri aslında son derece basittir. Amaç, kırık oluşurken meydana gelen yumuşak doku hasarına ek olarak, mümkün olan en az iyatrojenik hasarı vermek, yani minimal invaziv redüksiyon ve cerrahi teknikleri kullanarak ideal redüksiyonu sağlamak, ardından bu redüksiyonu koruyacak, 


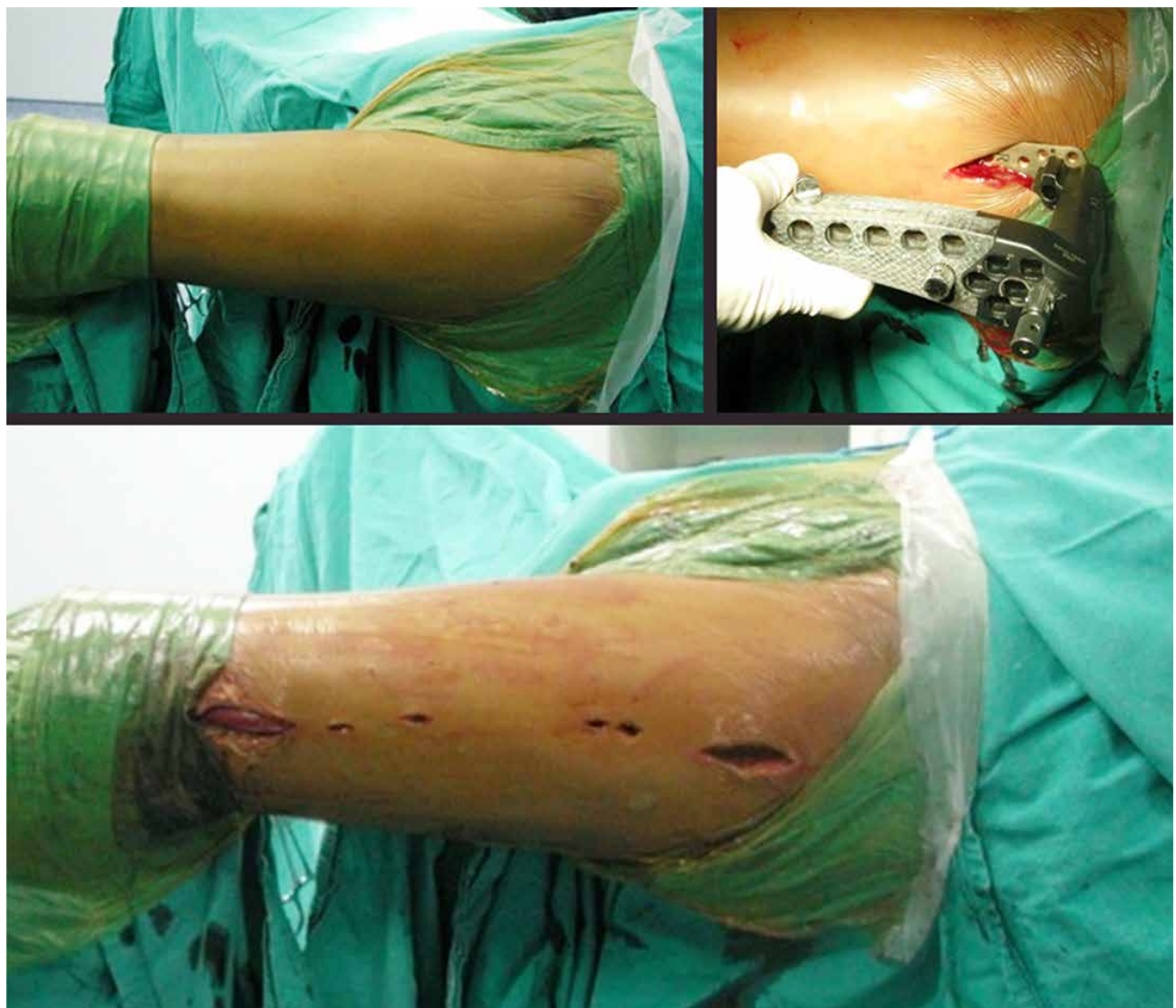

Şekil 17. Ters LISS plak ile minimal invaziv kırık tespiti.

stabiliteyi sağlayacak ideal implantı uygun cerrahi teknikle sağlamak ve olabildiğince erken hareket ile mobilizasyona izin vermektir. Proksimal femur kırıklarında kilitli intramedüller çivi yöntemi ilk tercih edilecek yöntem olarak kabul görse de $\mathrm{AO}$ prensiplerine uygun yapılan cerrahi girişimlerde plak-vida ile osteosentez yöntemi de iyi bir alternatif olabilir.

\section{KAYNAKLAR}

1. Cooper C, Campion G, Melton LJ 3rd. Hip fractures in the elderly: a world-wide projection. Osteoporos Int 1992;2(6):285-9. Crossref

2. Fixation using Alternative Implants for the Treatment of Hip fractures (FAITH) Investigators. Fracture fixation in the operative management of hip fractures (FAITH): an international, multicentre, randomised controlled trial. Lancet 2017;389(10078):1519-27. Crossref

3. Deangelis JP, Ademi A, Staff I, Lewis CG. Cemented versus uncemented hemiarthroplasty for displaced femoral neck fractures: a prospective randomized trial with early follow-up. J Orthop Trauma 2012;26(3):135-40. Crossref

4. Faldini C, Grandi G, Romagnoli M, Pagkrati S, Digennaro V, Faldini O, Giannini S. Surgical treatment of unstable intertrochanteric fractures by bipolar hip replacement or total hip replacement in elderly osteoporotic patients. J Orthop Traumatol 2006;7(3):117-21. Crossref
5. Geiger F, Zimmermann-Stenzel M, Heisel C, Lehner B, Daecke W. Trochanteric fractures in the elderly: the influence of primary hip arthroplasty on 1-year mortality. Arch Orthop Trauma Surg 2007;127(10):959-66. Crossref

6. Aktselis I, Kokoroghiannis C, Fragkomichalos E, Koundis G, Deligeorgis A, Daskalakis E, Vlamis J, Papaioannou N. Prospective randomised controlled trial of an intramedullary nail versus a sliding hip screw for intertrochanteric fractures of the femur. Int Orthop 2014;38(1):155-61. Crossref

7. Socci AR, Casemyr NE, Leslie MP, Baumgaertner MR. Implant options for the treatment of intertrochanteric fractures of the hip: rationale, evidence, and recommendations. Bone Joint J 2017;99-B(1):128-33. Crossref

8. Egol KA, Marcano Al, Lewis L, Tejwani NC, McLaurin TM, Davidovitch RI. Can the use of an evidence-based algorithm for the treatment of intertrochanteric fractures of the hip maintain quality at a reduced cost? Bone Joint J 2014;96$B(9): 1192-7$. Crossref

9. Marmor M, Liddle K, Buckley J, Matityahu A. Effect of varus and valgus alignment on implant loading after proximal femur fracture fixation. Eur J Orthop Surg Traumatol 2016;26(4):379-83. Crossref

10. Bhandari $M$, Schemitsch $E$, Jönsson A, Zlowodzki M, Haidukewych GJ. Gamma nails revisited: gamma nails versus compression hip screws in the management of intertrochanteric fractures of the hip: a meta-analysis. J Orthop Trauma 2009;23(6):460-4. Crossref 
11. Saudan M, Lübbeke A, Sadowski C, Riand N, Stern R, Hoffmeyer P. Pertrochanteric fractures: is there an advantage to an intramedullary nail?: a randomized, prospective study of 206 patients comparing the dynamic hip screw and proximal femoral nail. J Orthop Trauma 2002;16(6):386-93. Crossref

12. Parker MJ, Bowers TR, Pryor GA. Sliding hip screw versus the Targon PF nail in the treatment of trochanteric fractures of the hip: a randomised trial of 600 fractures. J Bone Joint Surg Br 2012;94-B(3):391-7. Crossref

13. Reindl R, Harvey EJ, Berry GK, Rahme E; Canadian Orthopaedic Trauma Society (COTS). Intramedullary Versus Extramedullary Fixation for Unstable Intertrochanteric Fractures: A Prospective Randomized Controlled Trial. J Bone Joint Surg Am 2015;97(23):1905-12. Crossref

14. Koh A, Guerado E, Giannoudis PV. Atypical femoral fractures related to bisphosphonate treatment: issues and controversies related to their surgical management. Bone Joint J 2017;99$B(3): 295-302$. Crossref

15. Lunsjö K, Ceder L, Thorngren KG, Skytting B, Tidermark J, Berntson PO, Allvin I, Norberg S, Hjalmars K, Larsson S, Knebel R, Hauggaard A, Stigsson L. Extramedullary fixation of 569 unstable intertrochanteric fractures: a randomized multicenter trial of the Medoff sliding plate versus three other screw-plate systems. Acta Orthop Scand 2001;72(2):13340. Crossref

16. Audigé L, Hanson B, Swiontkowski MF. Implant-related complications in the treatment of unstable intertrochanteric fractures: meta-analysis of dynamic screw-plate versus dynamic screw-intramedullary nail devices. Int Orthop 2003;27(4):197-203. Crossref

17. Krettek C, Müller M, Miclau T. Evolution of minimally invasive plate osteosynthesis (MIPO) in the femur. Injury 2001;32 Suppl 3:14-23. Crossref
18. Ozkaya U, Bilgili F, Kilic A, Parmaksizoglu AS, Kabukcuoglu Y. Minimally invasive management of unstable proximal femoral extracapsular fractures using reverse LISS femoral locking plates. Hip Int 2009;19(2):141-7. Crossref

19. Ma CH, Tu YK, Yu SW, Yen CY, Yeh JH, Wu CH. Reverse LISS plates for unstable proximal femoral fractures. Injury 2010;41(8):827-33. Crossref

20. Cornell CN, Ayalon O. Evidence for success with locking plates for fragility fractures. HSS J 2011;7(2):164-9. Crossref

21. Perren SM. Evolution of the internal fixation of long bone fractures. The scientific basis of biological internal fixation: choosing a new balance between stability and biology. J Bone Joint Surg Br 2002;84(8):1093-110. Crossref

22. Stoffel KK, Leys T, Damen N, Nicholls RL, Kuster MS. A new technique for cement augmentation of the sliding hip screw in proximal femur fractures. Clin Biomech (Bristol, Avon) 2008;23(1):45-51. Crossref

23. Haidukewych GJ. Intertrochanteric fractures: ten tips to improve results. J Bone Joint Surg Am 2009;91(3):712-9. https://www.ortopaedi.dk/fileadmin/specialespecifikke_ kurser/traumatologi/2013/litteratur/Intertrochanteric_ Fractures_-_Ten_Tips_to_Improve_Results.pdf

24. Kaufer $\mathrm{H}$. Mechanics of the treatment of hip injuries. Clin Orthop Relat Res 1980;(146):53-61. https://pubmed.ncbi. nlm.nih.gov/7371269/

25. CarrJB. The anterior and medial reduction of intertrochanteric fractures: a simple method to obtain a stable reduction. J Orthop Trauma 2007;21(7):485-9. Crossref 